\title{
Definitive Fassung der Richtlinien und Empfehlungen «Behandlung und Betreuung von älteren, pflegebedürftigen Menschen»
}

\author{
A. Stuck ${ }^{a}$
}

Die Subkommission «Behandlung und Betreuung von älteren, pflegebedürftigen Menschen» war überrascht und erfreut über die Zahl und die Qualität der eingegangenen Kommentare. Sie hat alle Stellungnahmen sorgfältig geprüft und wenn immer möglich berücksichtigt.

\section{Redaktionelle Kommentare}

Der Text wurde zum Teil als schwer verständlich beurteilt, und die Aufteilung in Richtlinie und Empfehlungen führte gelegentlich zu Unklarheiten.

\section{Antwort}

Wir haben den Text redaktionell vollständig überarbeitet. Unter anderem haben wir in den revidierten Richtlinien und Empfehlungen einheitlich die wichtigsten ethischen Diskussionspunkte hervorgehoben, um das Dokument damit benutzerfreundlicher zu gestalten.

\section{Geltungsbereich der Richtlinie}

Verschiedene Personen forderten, dass die Richtlinien nicht nur für ältere pflegebedürftige Per- sonen, sondern auch für jüngere pflegebedürftige Personen Gültigkeit haben sollte. Ebenso bemängelten mehrere Personen, dass die Adressaten der Richtlinien und Empfehlungen $\mathrm{zu}$ wenig klar oder umfassend beschrieben worden seien.

\section{Antwort}

Auch wenn es richtig ist, dass viele Punkte der vorliegenden Richtlinien ebenso für jüngere Personen Gültigkeit haben, gibt es dennoch Aspekte (z. B. Auswirkungen einer Pflegebedürftigkeit auf die berufliche Tätigkeit oder Behinderungen im Zusammenhang mit Geburtsgebrechen), bei denen sich zusätzliche oder andere ethische Herausforderungen ergeben. Zudem wirkten an der Entwicklung und Vernehmlassung primär Kreise mit, die Erfahrungen aus dem Altersbereich mitbringen. Aus diesem Grund lehnen wir die Erweiterung der Richtlinien auf jüngere Personen ab. Auf der andern Seite befürworten wir die klare Umschreibung von Zielgruppe und Geltungsbereich und haben deshalb im revidierten Text die Zielgruppe (Präambel) und den Geltungsbereich (separate Unterkapitel) klarer beschrieben. a Andreas Stuck ist Präsident der SAMW-Subkommission, welche diese Richtlinien erarbeitet hat. Er ist Chefarzt am Zentrum Geriatrie-Rehabilitation Spital Bern Ziegler und Honorarprofessor für Geriatrie an der Universität Bern.

1 Schweizerische Akademie der Medizinischen Wissenschaften. Behandlung und Betreuung von älteren pflegebedürftigen Menschen. Schweiz Ärztezeitung 2003;84(24):1281-91.
Die Erstpublikation der Richtlinien und Empfehlungen «Behandlung und Betreuung von älteren, pflegebedürftigen Menschen» Mitte Juni 2003 in der Schweizerischen Ärztezeitung [1] löste ein grosses Echo aus: Im Rahmen der Vernehmlassung trafen rund 50 Stellungnahmen von Personen und Organisationen aus verschiedensten Bereichen und Fachdisziplinen ein. Die Rückmeldungen waren mehrheitlich positiv; viele Personen erwarten, dass sich diese neue nationale Richtlinie positiv auf die Qualität der Betreuung älterer, pflegebedürftiger Personen auswirken wird. Zum Teil wurden in der Vernehmlassung Änderungen vorgeschlagen oder Kritik angebracht. Diese Kommentare werden im nachfolgenden Beitrag vom Präsidenten der Subkommission, Prof. Andreas Stuck aus Bern, zusammengefasst und beantwortet.

Im Namen der SAMW möchte ich an dieser Stelle der Subkommission und speziell ihrem Präsidenten für ihre grosse und wertvolle Arbeit herzlich danken. Gerne hoffe ich, dass die neuen Richtlinien und Empfehlungen die gebührende Aufmerksamkeit finden werden. Ein hoffnungsvolles Indiz in diese Richtung ist die Tatsache, dass auch der Schweizerische Berufsverband für Krankenpflege seinen Mitgliedern empfiehlt, die vorliegenden Richtlinien und Empfehlungen zu achten und anzuwenden.

Prof. Michel Vallotton, Präsident der Zentralen Ethikkommission (ZEK) der SAMW 


\section{Entscheidungsprozess bei fehlender Urteilsfähigkeit}

Von einigen Seiten wurde das in der Richtlinie vorgeschlagene Konzept der Vertrauensperson als unklar oder als juristisch problematisch dargestellt. Bei verschiedenen Aspekten (z. B. Hierarchie der Kompetenzen, Definition einer «Vertrauensperson») wurde bemängelt, dass der Prozess für die Praxis zu wenig genau beschrieben sei. Zudem wurde auf die Bedeutung der Richtlinie im Zusammenhang mit dem geplanten neuen Erwachsenenschutzgesetz hingewiesen.

\section{Antwort}

Wir haben die entsprechenden Kapitel der Richtlinien überarbeitet und den Prozess der Entscheidungsfindung noch präziser beschrieben. In diesem Zusammenhang präzisierten wir insbesondere die Definitionen der «Vertrauensperson» und das Vorgehen beim Vorliegen von Patientenverfügungen. Zudem klärten wir die Vorgehensweise für den Fall, dass keine bevollmächtigte vertretende Person vorhanden ist. Aufgrund einer aktualisierten juristischen Überprüfung erläutern wir die geltenden gesetzlichen Regelungen im Zusammenhang mit einer urteilsunfähigen älteren Person ohne gesetzlichen Vertreter in einer ausführlichen Fussnote.

\section{Beihilfe zum Suizid}

Die Thematik der Beihilfe zum Suizid führte zu vielen Kommentaren. Einigen Personen war aufgrund der Richtlinien die geltende gesetzliche Bestimmung unklar. Andere Personen fanden insbesondere die detaillierten Handlungsempfehlungen an die Institutionen inadäquat und forderten zum Teil eine klare Distanzierung von der Praxis der Beihilfe zum Suizid. Unbestritten war jedoch in den Stellungnahmen die Tatsache, dass die Aktivität von Sterbehilfeorganisationen bei pflegebedürftigen Personen besonders kritisch betrachtet werden muss.

\section{Antwort}

Der Text zur Beihilfe zum Suizid wurde überarbeitet. Dadurch, dass das Kapitel neu mit «Umgang mit dem Wunsch nach Beihilfe zum Suizid» genannt wird, besteht eine klare Abgrenzung zur Thematik Suizidalität im Sinne einer medizinischen Notfallsituation. Im revidierten Text wird der Schutzauftrag des Fachpersonals und des Heims genau beschrieben. Die grundlegenden Fragen im Zusammenhang mit der Beihilfe zum
Suizid werden im Rahmen der neuen Richtlinien «Betreuung von Patienten am Lebensende» geregelt.

\section{Datenschutz}

Konkrete Vorschläge gingen ein zu den Bereichen Datenschutz und Dokumentation. Es wurde gefordert, dass eine Richtlinie die ethischen Dimensionen des Datenschutzes aufzeigen und sich nicht auf die rechtlichen Aspekte beschränken solle.

\section{Antwort}

In den revidierten Richtlinien werden die Aspekte des Datenschutzes neu ausführlich erläutert. Dabei liessen wir uns von folgenden Prinzipien leiten: Die zu verwendenden, geriatrischen Assessmentinstrumente müssen auf ihre Verhältnismässigkeit und Aussagekraft überprüft worden sein, und die betroffenen alten Menschen müssen über die Tatsache der Informationssammlung und deren Zweck informiert sein. Als besonders schützenswerte Daten sind die Pflegedokumentationen und Krankengeschichten so zu handhaben und aufzubewahren, dass nur berechtigte Personen Einblick nehmen können.

\section{Praktikabilität der Richtlinie}

Viele Personen oder Stellen wiesen darauf hin, dass die Umsetzung der Richtlinien und Empfehlungen unrealistisch sei, wenn ein ausreichender Personalstellenetat nicht gewährleistet sei. Zum Teil wurde gefordert, dass finanzielle oder personelle Rahmenbedingungen formuliert werden sollten.

\section{Antwort}

Selbstverständlich ist es richtig, dass diese Aspekte massgeblich, wenn nicht hauptsächlich die Qualität der Behandlung und Betreuung älterer pflegebedürftiger Personen bedingen. Auf der andern Seite kann es nicht Aufgabe einer ethischen Richtlinie sein, hier für die Praxis verbindliche Normwerte zu definieren. Diese Kommentare haben uns aber gezeigt, dass es zum Teil nicht klar war, ob es sich bei den vorgelegten Richtlinien um Hinweise für die heutige Praxis oder um Idealvorstellungen für eine ferne $\mathrm{Zu}$ kunft handelt. Deshalb haben wir in der Präambel prägnant festgehalten, dass die Richtlinien praktikable Hinweise (nicht Idealvorstellungen) für die Praxis geben. 


\section{Weitere Vorschläge}

An verschiedenen Stellen forderten Personen oder Organisationen zusätzliche Klärungen oder genaue Hinweise. So zum Beispiel, welche Therapien wann angeboten werden müssen oder wie die Dokumentation genau geregelt sein soll.

\section{Antwort}

Die Richtlinie formuliert gut begründbare konzeptuelle Rahmenbedingungen und verzichtet auf möglicherweise umstrittene Detaillierungen. Einige Kommentare, wie zum Beispiel das klarere Ansprechen spiritueller Aspekte oder die durchgehende Erwähnung der Therapeuten, sind in die revidierten Richtlinien eingeflossen.

\section{Behandlung und Betreuung von älteren, pflegebedürftigen Menschen}

Medizinisch-ethische Richtlinien und Empfehlungen

Die deutsche Fassung

ist die Stammversion.

1 Aus Gründen der leichteren Lesbarkeit gilt in diesem Text die männliche Bezeichnung für beide Geschlechter.

2 Physiotherapeuten, Ergotherapeuten, Aktivierungstherapeuten, Logopäden, Psychologen.

3 Die Richtlinien der SAMW richten sich an medizinische Fachpersonen (Ärzte, Pflegende und Therapeuten) und haben im Prinzip einen verbindlichen Charakter; dies gilt besonders für Ärzte, da die Richtlinien in der Regel in die Standesordnung der $\mathrm{FMH}$ aufgenommen werden.

4 Da die SAMW Institutionen der Langzeitpflege gegenüber keine Regelungskompetenz hat, werden statt Richtlinien lediglich «Empfehlungen» formuliert.

\section{Präambel}

Die demographische Entwicklung in der Schweiz führt dazu, dass in den nächsten Jahren die Anzahl älterer, vor allem hochbetagter Personen deutlich ansteigen wird. Voraussichtlich wird deshalb die Anzahl pflegebedürftiger Personen ebenfalls markant zunehmen. Dies geschieht in einer Zeit des Wandels traditioneller Familienstrukturen, in einer Zeit, in der sich die Wertvorstellungen stark verändern und der Autonomie des Individuums eine immer grössere Bedeutung zukommt, in einer Zeit auch mit steigenden Gesundheitskosten.

Alle diese Faktoren führen dazu, dass die Behandlung und die Betreuung älterer, pflegebedürftiger Menschen mit verschiedenen Spannungsfeldern verbunden sind. Es kann einen Konflikt geben zwischen der notwendigen Fürsorge und der Respektierung der Autonomie einer älteren Person. Oft besteht ein Dilemma zwischen der notwendigen Aktivierung einer älteren Person und ihrem Wunsch nach Ruhe. Wann ist es angebracht, eine Krankheit therapeutisch anzugehen, und wann, auf kurative Interventionen $\mathrm{zu}$ verzichten? Vor allem in Institutionen der Langzeitpflege ergibt sich zudem das Spannungsfeld Privatheit versus Öffentlichkeit, stellt doch eine Institution gleichzeitig den privaten Wohnbereich der älteren Person und eine kollektive Betreuungsform dar. Die Diskussion um die Kosten im Gesundheitswesen hat die Herausforderungen bei der Behandlung und Betreuung älterer, pflegebedürftiger Personen zusätzlich akzentuiert.
Aus diesen Überlegungen verfolgt der nachfolgende Text drei Zielsetzungen: Erstens stellt er klar, dass Alter und Pflegebedürftigkeit nicht zur Vorenthaltung indizierter Massnahmen führen dürfen; zweitens bietet er Ärzten ${ }^{1}$, Pflegenden und Therapeuten ${ }^{2}$ in den Richtlinien ${ }^{3}$ eine Hilfe für Entscheidungen in schwierigen Situationen; drittens zeigt er in den Empfehlungen ${ }^{4}$ die wichtigen Anforderungen und Rahmenbedingungen für eine gute Behandlung und Betreuung älterer, pflegebedürftiger Personen auf.

Angesprochen sind aber auch Institutionen der Aus-, Weiter- und Fortbildung sowie politische Instanzen: Sie sind aufgefordert, in ihren Entscheiden im Bereich der Behandlung und Betreuung älterer, pflegebedürftiger Menschen den vorliegenden Richtlinien und Empfehlungen Rechnung zu tragen.

Ausdrücklich ist die Behandlung und Betreuung jüngerer, pflegebedürftiger Personen nicht Gegenstand dieser Richtlinien. Bei ihnen sind zusätzliche spezifische Aspekte zu beachten.

\section{Richtlinien (für Ärzte, Pflegende und Therapeuten)}

\section{Geltungsbereich}

Die vorliegenden Richtlinien richten sich an Ärzte, Pflegende und Therapeuten, welche ältere, pflegebedürftige Personen betreuen, sei dies zu Hause, in Spitälern oder in Institutionen der Langzeitpflege. Von einer «älteren Person» spricht man bei einem Menschen jenseits des 65. Lebensjahres; «Pflegebedürftigkeit» bedeutet 
das dauernde Angewiesensein auf Hilfe oder Unterstützung in grundlegenden Aktivitäten des täglichen Lebens (d.h. sich ankleiden, Körperpflege, Nahrungszufuhr, Benutzung der Toilette, Mobilität, Gestaltung des Tagesablaufs, soziale Kontakte). Die Pflegebedürftigkeit steigt in der Regel erst jenseits des 75. Lebensjahres markant an.

\section{Grundsätze}

\subsection{Angemessene Betreuung}

Ältere, pflegebedürftige Menschen haben bis an ihr Lebensende Anspruch auf eine angemessene Behandlung und Betreuung. Alter und Pflegebedürftigkeit einer betreuten Person dürfen nicht zu einer Vorenthaltung indizierter Massnahmen führen. Der behandelnde Arzt, das Pflegepersonal und die Therapeuten stützen ihre Entscheide auf eine gemeinsame Evaluation medizinischer, psychischer, sozialer und funktionaler Aspekte und des Umfelds. Sie respektieren bei der Betreuung die Würde, die Privatsphäre und die Intimsphäre der älteren Person, auch dann, wenn diese nicht mehr urteilsfähig ist oder unter psychischen Störungen leidet.

\subsection{Persönliche und kontinuierliche Betreuung}

Für eine adäquate Betreuung ist ein persönlicher Kontakt zwischen dem Arzt und der älteren, pflegebedürftigen Person unabdingbar. Bei älteren, pflegebedürftigen Personen kann es durch den Wechsel der Lebensorte (Zuhause, Spital, Institution der Langzeitpflege) zu einem Wechsel der ärztlichen Zuständigkeit kommen. Ärzte, welche eine ältere, pflegebedürftige Person in einem Spital oder in einer Institution der Langzeitpflege betreuen, haben sich so zu organisieren, dass jederzeit Klarheit darüber besteht, bei wem die ärztliche Zuständigkeit liegt; sie haben die ältere Person (oder im Fall der Urteilsunfähigkeit deren Vertrauensperson [siehe 3.3] bzw. gesetzlichen Vertreter) entsprechend $\mathrm{zu}$ informieren. Bei einem Wechsel der ärztlichen Zuständigkeit sind die beteiligten Ärzte dafür besorgt, dass der zuständige Arzt über alle für die weiterführende Betreuung erforderlichen Informationen verfügt.

An der Pflege einer älteren Person sind oft verschiedene Fachpersonen beteiligt, was es für die betreute Person schwierig macht, die für die Pflege bzw. Koordination verantwortliche Fachperson zu kennen. Im Spitexbereich, in Spitälern und in Institutionen der Langzeitpflege bezeichnet das Pflege- und Therapeutenteam für jeden älteren Patienten eine qualifizierte Ansprech- person und informiert die betreute Person und ihre Bezugspersonen entsprechend.

\subsection{Zusammenarbeit mit dem sozialen Umfeld}

Für Aspekte der Behandlung oder Betreuung pflegen der behandelnde Arzt und die Ansprechperson der Pflege wenn möglich mit dem sozialen Umfeld der betreuten Person (Lebenspartner, Verwandte und enge Bezugspersonen) guten Kontakt. Solche Kontakte unterliegen selbstverständlich dem Einverständnis der urteilsfähigen, älteren Person sowie den Regeln des Berufsgeheimnisses bzw. der Vertraulichkeit.

Bei der Betreuung von älteren, pflegebedürftigen Menschen zu Hause übernehmen Angehörige einen grossen Teil der Aufgaben; dies kann zu grossen Belastungen führen. Ärzte, Pflegende und Therapeuten haben die Aufgabe, die Angehörigen oder andere betreuende Personen zu beraten und zu unterstützen.

\subsection{Interdisziplinäre Zusammenarbeit}

Bei der Betreuung und Behandlung von älteren, pflegebedürftigen Menschen sind Ärzte, Pflegende, Therapeuten und zahlreiche andere Personen bzw. Berufsgruppen involviert. Aus diesem Grund ist es notwendig, dass Ärzte, Pflegende und Therapeuten systematisch und in dafür geeigneten Strukturen miteinander und mit den weiteren beteiligten Berufsgruppen zusammenarbeiten. In Institutionen der Langzeitpflege ist dabei auch auf die Zusammenarbeit mit dem Haus-, Küchen- und Verwaltungspersonal zu achten, dies unter Beachtung der Regeln des Berufsgeheimnisses bzw. der Vertraulichkeit.

\subsection{Angemessene Aus-, Weiter- und Fortbildung} Ältere, pflegebedürftige Personen leiden oft gleichzeitig an mehreren, vielfach chronischen Krankheiten (Multimorbidität). Zusätzlich spielen bei der Betreuung psychische, soziale, spirituelle und umgebungsbezogene Faktoren eine wichtige Rolle. Dies erfordert von den betreuenden Ärzten, Pflegenden und Therapeuten spezifische Kompetenzen in Geriatrie, Gerontologie und Alterspsychiatrie. $\mathrm{Zu}$ diesen Kompetenzen gehören insbesondere auch das Erheben des Gesundheitszustandes durch ein multidimensionales Assessment und die Einleitung, Durchführung und Evaluation geeigneter Massnahmen.

Ärzte, Pflegende und Therapeuten, welche ältere, pflegebedürftige Personen betreuen, sind verpflichtet, diese Kompetenzen durch Aus-, Weiter- und Fortbildung $\mathrm{zu}$ erwerben und $\mathrm{zu}$ erweitern. 


\section{Entscheidungsprozesse}

\subsection{Grundsatz}

Der Anspruch auf Respektierung der Menschenwürde und Autonomie gilt uneingeschränkt für alle Menschen. Das Recht materialisiert diesen Grundanspruch als das Recht auf «Respektierung der Menschenwürde», auf «Schutz der Persönlichkeit» und auf «Selbstbestimmung».

Eingeschränkte Autonomiefähigkeiten, welche mit zunehmendem Alter häufiger werden und das Gleichgewicht zwischen den abhängigen und unabhängigen Seiten bei einem Menschen stören, heben den Anspruch auf Respektierung seiner Würde und Autonomie nicht auf. Deshalb sind verbindliche Entscheidungsverfahren und Strukturen erforderlich, die einen Entscheidungsprozess unter Berücksichtigung der Selbstbestimmung und Würde des älteren Menschen ermöglichen. Dabei soll besonders darauf geachtet werden, dass die ältere Person ihren Willen äussern kann, dass sie den Umständen entsprechend ausreichend Zeit für wichtige Entscheidungen hat und dass sie Entscheidungen ohne Druck fällen kann.

\subsection{Patientenverfügung}

Jede Person kann im voraus Bestimmungen verfassen im Hinblick auf die medizinische Behandlung und Pflege, die sie zu erhalten wünscht oder ablehnt, falls sie nicht mehr urteilsfähig wäre. Falls die Voraussetzungen der Urteilsfähigkeit gegeben sind, können solche Patientenverfügungen von ihrem Verfasser jederzeit geändert oder aufgehoben werden.

Ärzte und Pflegende machen ältere Personen auf die Möglichkeit einer Patientenverfügung und deren regelmässig notwendige Aktualisierung aufmerksam; sie sprechen untereinander $\mathrm{ab}$, wer diese Aufgabe übernimmt.

\subsection{Bevollmächtigte Vertretungsperson in medizinischen Angelegenheiten}

Jede Person kann im voraus eine bevollmächtigte Vertretungsperson in medizinischen Angelegenheiten (nachstehend: «Vertrauensperson») festlegen, die an ihrer Stelle die Zustimmung zu medizinischen, pflegerischen und/oder therapeutischen Massnahmen erteilen soll, falls sie selbst nicht mehr urteilsfähig wäre. Ärzte und Pflegende machen ältere Personen frühzeitig auf die Möglichkeit der Bezeichnung einer Vertrauensperson und die regelmässig notwendige Aktualisierung dieser Bevollmächtigung aufmerksam; sie sprechen untereinander $a b$, wer diese Aufgabe übernimmt.

\subsection{Erarbeiten von Entscheidungsgrundlagen im Team}

Verschiedene Massnahmen wie die Behandlung einer Verhaltensstörung, die Dekubitusbehandlung oder die Einlage einer Nährsonde erfordern oft einen interdisziplinären Entscheidungsprozess. Bevor der behandelnde Arzt der älteren Person eine solche Massnahme vorschlägt und diese danach bei deren Einverständnis verordnet, bespricht er sie mit den zuständigen Ansprechpersonen der Pflege und Therapie und berücksichtigt deren Meinung.

Ebenso verlangt die Lösung komplexer Situationen (z.B. Fragen der Zukunftsplanung, Beratung von Angehörigen, Probleme des Zusammenlebens in einem Heim) oft einen interdisziplinären Entscheidungsprozess, der sich am Willen der älteren Person orientiert und dabei deren Vorstellungen, Ziele, Wünsche und Bedürfnisse miteinbezieht. Solche Situationen sind von den Beteiligten gemeinsam zu besprechen; Lösungsmöglichkeiten und korrigierende Massnahmen sind gemeinsam zu vereinbaren, bevor sie der älteren Person durch die zuständige Fachperson vorgeschlagen werden.

Die Notwendigkeit der interdisziplinären Zusammenarbeit entbindet die behandelnden Ärzte, Pflegenden und Therapeuten nicht von ihrer Verantwortung in bezug auf Entscheidungen und Massnahmen in ihrem beruflichen Zuständigkeitsbereich.

\subsection{Information}

Die ältere, pflegebedürftige Person hat Anspruch, durch den Arzt, die zuständige Person der Pflege oder den Therapeuten über vorgesehene diagnostische, präventive, pflegerische oder therapeutische Massnahmen informiert zu werden, damit sie den Massnahmen frei und aufgeklärt zustimmen kann. Die Information muss in geeigneter Weise gegeben werden, d.h. verständlich, differenziert - mit allfälligen Entscheidvarianten - und der Situation angepasst. $\mathrm{Zu}$ jeder Variante sind Nutzen und Risiken zu formulieren. Nach Möglichkeit und falls die ältere Person damit einverstanden ist, soll auch ihre Vertrauensperson oder eine ihr nahestehende Person informiert werden, damit sie die ältere Person in ihrem Entscheidprozess unterstützen kann.

Falls die ältere Person urteilsunfähig ist, erhält ihre Vertrauensperson bzw. ihr gesetzlicher Vertreter diese Informationen; selbstverständlich soll die betroffene Person die Informationen in angemessener Form ebenfalls erhalten. 


\subsection{Einwilligung der urteilsfähigen, älteren Person $^{5}$}

Ärzte, Pflegende und Therapeuten dürfen eine Massnahme nur mit der freien Einwilligung der urteilsfähigen, informierten älteren Person durchführen.

Lehnt eine urteilsfähige, ältere Person die ihr vorgeschlagenen Massnahmen ab, nachdem sie über diese und die möglichen Folgen der Ablehnung informiert worden ist, so haben der Arzt und das Pflegepersonal diesen Entscheid zu respektieren. Falls dieser ablehnende Entscheid aus Sicht der verantwortlichen Fachpersonen nicht im besten Interesse der älteren Person liegt, so suchen sie nach einer anderen, für die betreffende Person voraussichtlich akzeptablen Behandlungsmöglichkeit.

\subsection{Einwilligungsverfahren bei Urteilsunfähigkeit der älteren Person}

Bei Urteilsunfähigkeit der älteren Person im Hinblick auf eine Entscheidung klärt der Arzt oder das Pflegepersonal ab, ob sie eine Patientenverfügung verfasst hat, ob sie eine Vertrauensperson bevollmächtigt hat und/oder ob ein gesetzlicher Vertreter bezeichnet ist.

Patientenverfügungen sind zu befolgen, solange keine konkreten Anhaltspunkte dafür bestehen, dass sie dem derzeitigen Willen der betreffenden Person nicht mehr entsprechen.

Falls keine Patientenverfügung vorliegt oder wenn ein begründeter Zweifel darüber besteht, ob die Willensäusserung noch gültig ist, muss der Arzt in jedem Fall die Zustimmung der von der älteren Person bezeichneten Vertrauensperson bzw. des (allenfalls neu zu bestimmenden) gesetzlichen Vertreters einholen. Jeder Entscheid soll sich am mutmasslichen Willen der urteilsunfähigen, älteren Person orientieren und in ihrem besten Interesse getroffen werden. Falls der Entscheid der Vertrauensperson bzw. des gesetzlichen Vertreters dem mutmasslichen Willen der älteren Person zu widersprechen scheint, hat der Arzt die Vormundschaftsbehörde zu kontaktieren.

Gibt es bei fehlender Patientenverfügung weder Vertrauensperson (oder lehnt diese den Vorsorgeauftrag ab bzw. ist nicht in der Lage, diesen wahrzunehmen) noch gesetzlichen Vertreter oder ist in einer Notfallsituation eine Rückfrage nicht möglich, haben der Arzt, die Pflegenden und Therapeuten ihre Entscheide im interdisziplinären Austausch, gemäss den objektiven Interessen und dem mutmasslichen Willen der betroffenen Person zu treffen - dies unter der Voraussetzung, dass keine anderslautenden kantonalen Vorschriften bestehen. ${ }^{6}$ Das soziale
Umfeld (Lebenspartner, Verwandte und enge Bezugspersonen) ist wenn immer möglich in diesen Entscheidungsprozess miteinzubeziehen.

\section{Behandlung und Betreuung}

\subsection{Gesundheitsförderung und Prävention}

Es ist ärztliche, pflegerische und therapeutische Aufgabe, der älteren, pflegebedürftigen Person Massnahmen vorzuschlagen und $\mathrm{zu}$ ermöglichen, die ihr erlauben, ihre physischen, psychischen und sozialen Kompetenzen und Ressourcen $\mathrm{zu}$ erhalten oder $\mathrm{zu}$ fördern. Pflegebedürftige ältere Menschen sind besonders häufig bestimmten Risiken (z.B. Sturz, Immobilität, Depression, Ernährungsstörungen, Wundliegen, Erleiden von Gewalt, Misshandlung) ausgesetzt. Es ist ärztliche, pflegerische und therapeutische Aufgabe, diese frühzeitig zu erkennen und, nach Information und Zustimmung der älteren Person, die zweckmässigen präventiven Massnahmen zu ergreifen.

\subsection{Akuttherapie}

Es ist ärztliche, pflegerische und therapeutische Aufgabe, sicherzustellen, dass ältere, pflegebedürftige Menschen bei akuter Erkrankung Zugang zu einer adäquaten Abklärung und Behandlung haben. Dabei ist auch im Akutspital die durch die Pflegebedürftigkeit bedingte spezifische Betreuung (z.B. bei Demenz, Dekubitus oder Inkontinenz) zu gewährleisten.

\subsection{Rehabilitation}

Es ist ärztliche, pflegerische und therapeutische Aufgabe, der älteren, pflegebedürftigen Person jene Behandlung (u.a. soziale Kontakte, Physiotherapie, Psychotherapie, Ergotherapie, Logopädie, zahnärztliche Behandlung, Versorgung mit Hörgeräten) und Betreuung (u.a. soziale Kontakte, Ernährung, Mobilisation, Aktivitäten, Tagesstruktur) vorzuschlagen und zu ermöglichen, die ihr erlauben, ihre physischen und psychischen und sozialen Kompetenzen und Ressourcen soweit als möglich zu erhalten oder wiederzuerlangen.

\subsection{Palliative Betreuung}

Der Zugang zu palliativer Medizin, Pflege und Betreuung ist allen älteren, pflegebedürftigen Menschen rechtzeitig zu garantieren, unabhängig vom Ort, wo sie leben. Sowohl in Institutionen der Langzeitpflege als auch in der ambulanten Krankenpflege oder im Spital kennen die Ärzte, Pflegenden und Therapeuten die Konzepte palliativer Betreuung und wenden sie an. Der Arzt, die Pflegenden und die Therapeu- 
6 Die Behandlung des urteilsunfähigen Patienten, welcher keinen gesetzlichen Vertreter hat und auch keine Vertrauensperson bezeichnet hat, ist auf eidgenössischer Ebene nicht ausdrücklich geregelt. Hingegen existieren auf kantonaler Ebene entsprechende gesetzliche Regelungen; diese sind jedoch uneinheitlich. Einige räumen dem Arzt unter gewissen Umständen ein Entscheidungsrecht ein (so z.B. Aargau, Appenzell A. Rh., Bern, Luzern, Thurgau, Zürich [Stand 2003]). Andere Kantone sehen in einer solchen Situation eine Vertretungsvollmacht der Familie oder enger Bezugspersonen vor (z.B. Neuenburg, Jura, Tessin). Wieder andere verlangen vom Arzt, dass er bei der Vormundschaftsbehörde um die Bezeichnung eines gesetzlichen Vertreters nachsucht (z.B. Genf). Urteilsfähige, ältere, pflegebedürftige Personen in Institutionen der Langzeitpflege haben heutzutage häufig keinen Vertreter in medizinischen Angelegenheiten. Im Hinblick auf eine mittelfristige Umsetzung des Prinzips der Einwilligung eines Vertreters bei Urteilsunfähigkeit (wie sie z.B. auch die zur Ratifikation vorgesehene Biomedizinkonvention vorsieht) formuliert diese Richtlinie bewusst die Forderung, dass Ärzte, Pflegende und Therapeuten ihre Patienten frühzeitig dazu anregen, eine bevollmächtigte «Vertrauensperson» zu bestimmen. Ausserdem wird allen Ärzten geraten, sich im Zweifelsfall bei der zuständigen Vormund schaftsbehörde juristisch beraten zu lassen.

7 Die Pflegedokumentation erfüllt folgende Zwecke:

- stellt die Patientensituation aus pflegerischer Sicht (Assessments) dar;

- hält die für die Situation wesentlichen pflegerischen Interventionen und deren Evaluation fest;

- ermöglicht es, die Pflege nachzuvollziehen.

8 z.B. medikamentöse Ruhigstellung, Einschränkungen der Bewegungsfreiheit durch Gurten oder andere Freiheitseinschränkungen wie z.B. individuelles Rauchverbot. Die medikamentöse Ruhigstellung gilt in einigen Kantonen als medizinische Zwangsmassnahme und unterliegt deshalb besonderen Regelungen. ten nehmen insbesondere belastende Symptome wie Schmerzen, Angst, Depression und Hoffnungslosigkeit wahr und behandeln sie umfassend, dies unter Einbezug der Angehörigen. Die palliative Betreuung ist ein interdisziplinärer Prozess; bei Bedarf und auf Wunsch der älteren, pflegebedürftigen Person ist ein Seelsorger beizuziehen.

\section{Sterben und Tod}

\subsection{Begleitung von Sterbenden}

Die Begleitung und Betreuung von Sterbenden ist in den medizinisch-ethischen Richtlinien der Schweizerischen Akademie der Medizinischen Wissenschaften «Betreuung von Patienten am Lebensende» geregelt.

\subsection{Umgang mit dem Wunsch nach Suizid}

Äussert eine ältere, pflegebedürftige Person den Wunsch nach Selbsttötung, sucht das betreuende Team das Gespräch mit der betreffenden Person. In jedem Fall leiten der Arzt und das Pflegepersonal Massnahmen zum bestmöglichen Schutz und zur Unterstützung der betreffenden Person ein. Insbesondere klären sie mögliche Verbesserungen der Therapie-, Pflegeund Betreuungssituation. Dabei sind auch die vielfältigen Abhängigkeiten der älteren, pflegebedürftigen Person, die das Risiko einer Suizidalität erhöhen können, zu beachten. Das betreuende Team stellt sicher, dass die erforderlichen palliativen, therapeutischen und/oder psychiatrischen Massnahmen vorgeschlagen bzw. durchgeführt werden, ebenso, dass ein seelsorgerlicher Beistand vorgeschlagen und, falls gewünscht, vermittelt wird.

\section{Dokumentation und Datenschutz}

\subsection{Krankengeschichte und Pflegedokumentation}

Der Arzt führt über jede ältere, pflegebedürftige Person, die er betreut, eine Krankengeschichte. In der Krankengeschichte hält der Arzt Angaben betreffend Anamnese, Untersuchungen, Untersuchungsergebnisse, Beurteilung, Massnahmen und Verlauf fest und legt darin medizinisch relevante Dokumente ab. Die Pflegenden führen eine Pflegedokumentation ${ }^{7}$. Die relevanten Aspekte der ärztlichen Dokumentation sind dem zuständigen Pflegepersonal und den Therapeuten zugänglich.

Die Therapeuten dokumentieren den therapeutischen Prozess (Beobachtungen bei der Erfassung, Zielsetzung und Planung, Evaluation der Massnahmen). Eine Zusammenstellung der wichtigsten Beobachtungen, Ziele und Resultate ist dem zuständigen Arzt und dem zuständigen Pflegepersonal zugänglich.

Die ältere Person bzw. bei deren Urteilsunfähigkeit ihre Vertrauensperson oder ihr gesetzlicher Vertreter haben das Recht, die Krankengeschichte und die Pflegedokumentation einzusehen und sich diese erläutern zu lassen; sie können Kopien davon verlangen.

Die Krankengeschichte und die Pflegedokumentation enthalten die aktuelle Version einer allfälligen Patientenverfügung, Angaben zur Vertrauensperson oder zu einem allfälligen gesetzlichen Vertreter sowie allfällige Protokolle von freiheitsbeschränkenden Massnahmen.

\subsection{Verschwiegenheitspflicht}

Der Arzt, das Pflegepersonal und die Therapeuten sind an das Berufsgeheimnis gebunden.

Die Erhebung, die Ablage, die Auswertung und die Weitergabe von Daten dürfen nur unter Beachtung der gesetzlichen Datenschutzbestimmungen erfolgen.

Die zu verwendenden geriatrischen Assessmentinstrumente müssen auf ihre Verhältnismässigkeit und Aussagekraft überprüft worden sein, und die betroffenen älteren Personen müssen über die Tatsache der Informationssammlung und deren Zweck informiert sein.

Als besonders schützenswerte Daten sind die Pflegedokumentation und die Krankengeschichte so $\mathrm{zu}$ handhaben und aufzubewahren, dass nur berechtigte Personen Einblick nehmen können. Für die elektronische Datenverarbeitung sind die hohen Anforderungen betreffend Zugriffsschutz und Sicherheit der Datenübertragung und -ablage zu beachten.

Die Daten dürfen nur nach vollständiger Anonymisierung für statistische und wissenschaftliche Zwecke verwendet werden. Die Weitergabe von nicht-anonymisierten Daten setzt die ausdrückliche Zustimmung der Betroffenen (bzw. bei deren Urteilsunfähigkeit ihrer Vertrauensperson oder ihres gesetzlichen Vertreters) voraus.

\section{Anwendung von freiheitsbeschränkenden Massnahmen}

\subsection{Grundsatz}

Verhaltensstörungen, Unruhe und Verwirrtheit älterer, pflegebedürftiger Personen können zu einer Gefährdung ihrer selbst und/oder von Drittpersonen oder zu einer schwerwiegenden Belästigung von Drittpersonen führen. Die Anwendung freiheitsbeschränkender Massnahmen ${ }^{8}$ zur Vermeidung solcher Gefährdungen stellt einen Eingriff in die Grundrechte der 
älteren Person dar. Solche Massnahmen führen ausserdem nicht immer zu einer Reduktion der Gefährdung, sondern können diese noch erhöhen. Eine freiheitsbeschränkende Massnahme muss deshalb grundsätzlich die Ausnahme bleiben.

\subsection{Bedingungen}

Eine freiheitsbeschränkende Massnahme darf, unter Vorbehalt anderslautender gesetzlicher Vorschriften, nur unter folgenden Bedingungen eingesetzt werden:

a) das Verhalten der Person gefährdet in erheblichem Masse ihre eigene Sicherheit oder Gesundheit oder diejenige anderer Personen oder beeinträchtigt in hohem Ausmass Ruhe und Wohlbefinden Dritter;

b) das beobachtete auffällige Verhalten ist nicht auf behebbare Ursachen zurückzuführen, wie z.B. Schmerz, Nebenwirkungen von Medikamenten oder zwischenmenschliche Spannungen;

c) andere, die persönliche Freiheit weniger beeinträchtigende Massnahmen haben versagt oder sind nicht möglich.

Eine freiheitsbeschränkende Massnahme wird vom Arzt, vom Pflegeteam und von den Therapeuten gemeinsam besprochen, bevor sie der älteren Person (bzw. bei Urteilsunfähigkeit ihrer Vertrauensperson oder ihrem gesetzlichen Vertreter) vorgeschlagen wird.

Die ältere Person bzw. ihre Vertrauensperson oder ihr gesetzlicher Vertreter müssen über den Zweck, die Art und die Dauer der Massnahme verständlich und angemessen informiert werden; gleichzeitig ist ihnen der Name der verantwortlichen Person mitzuteilen (siehe unten Ziffer 7.3).

Grundsätzlich darf eine freiheitsbeschränkende Massnahme nur mit Zustimmung der betroffenen älteren Person bzw. bei deren Urteilsunfähigkeit mit Zustimmung ihrer Vertrauensperson oder ihres gesetzlichen Vertreters ergriffen werden.

Ist eine Person urteilsunfähig und hat weder Vertrauensperson noch gesetzlichen Vertreter oder ist in einer Notfallsituation eine Rückfrage nicht möglich, haben der Arzt, die Pflegenden und allenfalls zuständige Therapeuten eine solche Massnahme in einem interdisziplinären Entscheidungsprozess im besten Interesse der betroffenen Person und unter Einbezug der Angehörigen gemäss den obigen Kriterien zu beschliessen. ${ }^{9}$ Von einer einzigen Fachperson gefällte, kurzfristige Entscheide sollen anschliessend gemäss diesem Prozedere neu entschieden werden.

\subsection{Schriftliche Protokollierung}

Ein Protokoll, das zumindest den Zweck, die Dauer und die Art jeder angewendeten Massnahme sowie den Namen der verantwortlichen Person und das Ergebnis der regelmässigen Neubeurteilungen enthält, wird in die Krankengeschichte und/oder in die Pflegedokumentation aufgenommen.

\subsection{Begleitmassnahmen}

Stets sollte bewusst sein, dass bei freiheitsbeschränkenden Massnahmen die Gefahr von Schädigungen besteht. Deshalb muss während der Dauer der Massnahme die bestmögliche Überwachung der betroffenen Person sichergestellt sein. Die Massnahme wird in regelmässigen Abständen evaluiert; die Häufigkeit richtet sich nach der Art der Massnahme. Die Massnahme wird beendet, sobald die Bedingungen nach Ziffer 7.2 nicht mehr erfüllt sind.

\section{Misshandlung und Vernachlässigung}

Ältere, pflegebedürftige Personen sind in besonderem Masse verletzlich und müssen vor jeder Form von Gewaltanwendung geschützt werden, sei dies körperliche oder psychische Gewalt, Machtmissbrauch oder Vernachlässigung. Alle Spuren von Gewaltanwendung, Missbrauch oder Vernachlässigung, die das betreuende Team bei einer älteren Person beobachtet, muss es sorgfältig in der Krankengeschichte und in der Pflegedokumentation dokumentieren und dabei die objektivierbaren klinischen Befunde (Grösse, Lokalisation, Aussehen usw.) festhalten. Pflegende und Therapeuten haben Spuren von Gewalt, die sie beobachten, dem behandelnden Arzt zu melden.

Der Arzt, die Pflegenden und die Therapeuten haben die notwendigen Schritte einzuleiten, um weitere Misshandlungen zu vermeiden. Falls notwendig und mit dem Einverständnis der älteren Person (bzw. bei Urteilsunfähigkeit mit dem Einverständnis der Vertrauensperson bzw. des gesetzlichen Vertreters) werden diese Informationen an die zuständige Behörde übermittelt. Wenn ein solches Einverständnis fehlt, aber es im Interesse der älteren Person liegt, müssen die zuständigen Behörden informiert werden.

\section{Eintritt in eine Institution der Langzeit- pflege}

Der Eintritt in eine Institution der Langzeitpflege verbunden mit der Aufgabe der bisherigen Wohnsituation soll nur dann erfolgen, wenn aufgrund fehlender ambulanter Betreuungsmöglichkeiten oder eines begrenzten Rehabilitationspotentials ein Verbleib zu Hause oder eine 
Rückkehr nach Hause nicht mehr im besten Interesse einer älteren Person ist. In gewissen Situationen kann ein frühzeitiger Eintritt in eine Institution der Langzeitpflege sinnvoll sein, z.B., wenn dadurch die soziale Integration der älteren Person gefördert werden kann.

Vor einem geplanten Eintritt in eine Institution der Langzeitpflege führt der zuständige Arzt ein geriatrisches multidimensionales Assessment durch. Im Spital erfolgen diese Abklärungen unter Einbezug des Pflegepersonals und der Therapeuten sowie nach Möglichkeit in Zusammenarbeit mit dem Hausarzt, der Spitex, der ambulanten Therapie und dem sozialen Umfeld (Lebenspartner, Verwandte und enge Bezugspersonen) der älteren, pflegebedürftigen Person. Der Arzt informiert die ältere Person (und gegebenenfalls Personen aus ihrem sozialen Umfeld) über das Ergebnis dieses Assessments und bespricht mit ihr die Notwendigkeit eines Eintritts in eine Institution der Langzeitpflege bzw. allfällige Alternativen.

\section{Empfehlungen (an Institutionen der Langzeitpflege)}

\section{Geltungsbereich}

Die nachstehenden Empfehlungen richten sich in erster Linie an die Leitungen von Institutionen der Langzeitpflege und an die Trägerschaft; sinngemäss gelten sie jedoch auch für andere Institutionen (Spitäler, Spitex), welche ältere, pflegebedürftige Personen behandeln und betreuen. Damit richten sich diese Empfehlungen auch an Ärzte, Pflegende und Therapeuten, sofern sie in diesen Institutionen Leitungsfunktionen innehaben.

Die nachfolgenden Empfehlungen formulieren die Rahmenbedingungen, die in diesen Institutionen für eine gute Behandlung und Betreuung älterer Personen erforderlich sind. Für jeden in den voranstehenden Richtlinien definierten Bereich sind solche Empfehlungen formuliert.

\section{Grundsätze}

Die Institution schützt und respektiert die Rechte der älteren Person.

Schutz der persönlichen Freiheit und der Würde Die ältere Person hat Anspruch darauf, dass ihre persönliche Freiheit respektiert wird. Sie hat Anspruch darauf, dass ihr mit Höflichkeit und Respekt begegnet und ihrer Würde, ihrem Wohlergehen und ihrer Individualität Rechnung getragen wird.

\section{Achtung der Privat- und der Intimsphäre}

Die Institution respektiert die Privat- und die Intimsphäre der älteren Person inklusive deren sexuelle Freiheit.

Das Zimmer (oder der Teil des Zimmers), das die ältere Person bewohnt, ist Teil ihrer Privatsphäre und muss als solche vom Personal der Institution respektiert werden. Die ältere Person kann das Zimmer (oder einen Teil davon) in Absprache mit der Institution nach ihren persönlichen Vorstellungen gestalten, insbesondere durch eigene Möbel oder Wandschmuck. Die ältere Person verfügt über einen abschliessbaren Schrank, in dem sie ihre persönlichen Sachen ablegen kann. Falls ein Zimmer durch mehrere Personen belegt ist, ergreift die Institution die notwendigen Massnahmen, damit die Privatsphäre und die Intimsphäre jeder Person gewährleistet sind.

Das Personal behandelt Beobachtungen aus der Privat- bzw. Intimsphäre der älteren Person oder Ereignisse, welche diese nur mit einem beschränkten Kreis von Personen (Freunde, Verwandte) teilen will, diskret und leitet diese nur weiter, falls dies zur Sicherstellung einer angemessenen Pflege und Betreuung notwendig ist.

\section{Aufrechterhaltung der sozialen Kontakte}

Die Institution unterstützt die Aufrechterhaltung und Pflege der Beziehungen, welche die ältere Person mit ihren Angehörigen und ihrem sozialen Umfeld hat. Sie informiert die Angehörigen über kulturelle Aktivitäten innerhalb der Institution und versucht, sie zu integrieren. Die Institution sorgt dafür, dass vertrauliche Gespräche und Begegnungen in einem ungestörten Rahmen möglich sind.

Die ältere Person hat das Recht darauf, externe Kontakte zu pflegen (Briefe, Besuche, Zeitungen, Telefon, Fernsehen, Internet usw.).

\section{Meinungs- und Glaubensfreiheit}

Die ältere Person ist frei in ihren Meinungsäusserungen, sofern diese nicht gegen die Rechte Dritter oder gegen gesetzliche Bestimmungen (z.B. Rassismusnorm) verstossen. Die Institution sorgt dafür, dass die geäusserten Meinungen respektiert werden.

Die Institution respektiert die Glaubensfreiheit der älteren Person und lässt die Ausübung von religiösen Riten oder Ausdrucksformen zu; diese dürfen jedoch andere Personen bzw. das Umfeld nicht einschränken.

\section{Versammlungsfreiheit}

Die Institution respektiert die Versammlungsfreiheit der älteren Person. Die Institution er- 
mutigt Zusammenkünfte ihrer Bewohner, soweit diese dazu in der Lage sind; sie stellt ihnen Räumlichkeiten zur Verfügung, damit sie sich versammeln können.

\section{Politische Rechte}

Die Institution sorgt dafür, dass die ältere Person ihre politischen Rechte frei ausüben kann. Sie stellt sicher, dass nicht eine andere Person diese an ihrer Stelle ausübt oder von ihrer Abhängigkeit profitiert, um sie zu beeinflussen.

\section{Mitbestimmung bei der Alltagsgestaltung}

Die Bewohner der Institution, welche sich dazu äussern können, werden zu Entscheiden betreffend Fragen des Tagesablaufes, des Zusammenlebens und betreffend gemeinsamer Veranstaltungen beigezogen. Die Institution regelt die Form der Mitbestimmung.

\section{Beschwerderecht}

Die Institution etabliert ein internes Verfahren, wie mit Beschwerden (zu medizinischen, pflegerischen und/oder administrativen Belangen) umgegangen wird. Als Beschwerdeführer können sowohl die ältere Person als auch ihr(e) Vertreter bzw. die Angehörigen auftreten.

Die Institution stellt sicher, dass die Beschwerden innert Kürze, sorgfältig, unter Beachtung der Vertraulichkeit und ohne Nachteil für den Beschwerdeführer behandelt werden. Falls die Beschwerde gerechtfertigt ist, ergreift die Institution die notwendigen Massnahmen. Falls die Institution die Beschwerde ablehnt, weist sie den Beschwerdeführer auf Rekursmöglichkeiten oder gegebenenfalls auf Ombuds- bzw. unabhängige Beschwerdestellen hin.

\section{Entscheidungsprozesse}

Im Rahmen des Eintrittsprozederes vergewissert sich die Institution, ob die ältere Person für den Fall der Urteilsunfähigkeit einen «Bevollmächtigten» bezeichnet hat, der ihre Interessen in administrativen (inkl. finanziellen) Angelegenheiten wahrnehmen kann, sowie eine «bevollmächtigte Vertretungsperson in medizinischen Angelegenheiten» («Vertrauensperson»), welche an ihrer Stelle über die zu erteilende Behandlung und Pflege zu entscheiden hat.

Ist dies nicht der Fall, rät die Institution der älteren Person, Personen ihrer Wahl eine solche Vollmacht zu erteilen; allenfalls unterstützt die Institution die ältere Person bei der Suche nach geeigneten Personen. Die Funktion des «Bevollmächtigten in administrativen Angelegenheiten» und der «Vertrauensperson in therapeutischen Angelegenheiten» können von der gleichen Person oder von zwei verschiedenen Personen wahrgenommen werden.

Die Institution hält die Namen des «Bevollmächtigten» und der «Vertrauensperson» im administrativen Dossier fest; sie stellt sicher, dass der Arzt, das Pflegepersonal und die Therapeuten über das Vorhandensein einer «Vertrauensperson» informiert sind.

\section{Behandlung und Betreuung}

\section{Sicherstellung einer adäquaten Behandlung und Betreuung}

Bevor die Institution jemanden aufnimmt, überprüft sie, ob die dem Gesundheitszustand und dem Abhängigkeitsgrad der betroffenen Person entsprechenden Betreuungsmöglichkeiten vorhanden sind und ob sie sowohl über das Personal als auch die Ausrüstung verfügt, welche für eine adäquate Behandlung und Betreuung notwendig sind.

\section{Qualitätssicherung}

Alle Institutionen, welche ältere, pflegebedürftige Personen behandeln und betreuen, weisen sich über ein umfassendes Qualitätsmanagement für die adäquate Behandlung und Betreuung aus.

\section{Qualifiziertes Personal}

Die Institution stellt sicher, dass die Fachpersonen entsprechend ihrer Funktion über eine Ausbildung verfügen, welche sie für ihre Aufgabe qualifiziert. Die Institution unterstützt und fördert auch die regelmässige Weiter- und Fortbildung des Personals, unter besonderer Berücksichtigung des problemzentrierten Lernens in interdisziplinären Teams.

Die Institution bezeichnet einen verantwortlichen Heimarzt, der für die Organisation der medizinischen Betreuung in der Institution zuständig ist und die hierfür notwendigen Kenntnisse besitzt. Sind in einer Institution mehrere Ärzte tätig, soll die Institution in Absprache mit diesen einen davon als verantwortlichen Heimarzt bezeichnen.

\section{Sterben und Tod}

\section{Begleitung von Sterbenden}

Die Begleitung der älteren Person am Lebensende soll unter Beachtung ihrer Bedürfnisse und ihrer Überzeugungen erfolgen. Die Institution achtet darauf, dass die ältere Person von ihrem sozialen Umfeld so viel wie möglich (und soviel wie von ihr gewünscht) unterstützt wird. Die sterbende Person soll ungestört und an einem 
geeigneten Ort von ihren Nächsten Abschied nehmen können und hat Anspruch auf spirituellen Beistand ihrer Wahl.

Die Institution schafft einen Rahmen, der Abschiedsrituale und -riten für alle Beteiligten sicherstellt. Die Institution respektiert besondere religiöse und kulturelle Abschiedsrituale der Hinterbliebenen.

\section{Umgang mit dem Wunsch nach Beihilfe zum Suizid}

Eine besondere Situation liegt dann vor, wenn eine ältere, pflegebedürftige Person in einer Institution der Langzeitpflege einen Suizid unter Beihilfe von Dritten (z.B. einer Sterbehilfeorganisation) plant. Diese Situation kann eintreten, weil gemäss schweizerischem Recht die Beihilfe zum Suizid nicht strafbar ist, ausser beim Vorliegen selbstsüchtiger Motive (Art. 115 StGB). Es gibt Institutionen, die auf dieser Grundlage die Beihilfe zum Suizid zulassen. In solchen Situationen ist zu beachten, dass eine Institution der Langzeitpflege besondere Schutzpflichten hat und daher folgendes beachten muss:

a) es muss sichergestellt sein, dass die betreffende Person urteilsfähig ist;

b) es muss sichergestellt sein, dass der Entscheid zum Suizid nicht auf äusseren Druck oder auf eine nicht adäquate Abklärung, Behandlung oder Betreuung zurückzuführen ist;

c) es muss sichergestellt sein, dass die Gefühle der Mitbewohner und der Mitarbeiter respektiert werden.

Ältere, pflegebedürftige Personen stehen in einem besonderen Abhängigkeitsverhältnis zum Personal der Institution; dieses Verhältnis kann beim Personal zu Interessenkonflikten führen. Aus diesem Grund und aus Rücksichtnahme auf die übrigen Bewohner der Institution soll das Personal einer Institution der Langzeitpflege zu keinem Zeitpunkt an der Durchführung eines Suizids mitwirken.

\section{Dokumentation und Datenschutz}

Die ältere Person (oder bei deren Urteilsunfähigkeit ihre Vertrauensperson oder ihr gesetzlicher Vertreter) kann das sie betreffende administrative Dossier konsultieren und sich erläutern lassen.

Die Institution respektiert die gesetzlichen Bestimmungen des Datenschutzes. Sie schenkt diesen besondere Aufmerksamkeit im Falle der elektronischen Datenverarbeitung (namentlich zum Zweck der Tarifbestimmung, der Qualitätssicherung oder Forschung).

\section{Anwendung von freiheitsbeschränkenden Massnahmen}

Die Institution stellt sicher, dass bei jeder freiheitsbeschränkenden Massnahme Ziffer 7 der vorliegenden Richtlinien erfüllt ist.

\section{Misshandlung und Vernachlässigung}

Die Institution stellt sicher, dass es nicht zur Misshandlung oder Vernachlässigung von älteren Personen kommt; sie sorgt dafür, dass die Ziffer 8 der vorliegenden Richtlinie bekannt ist und angewendet wird.

\section{Eintritt in eine Institution der Langzeit- pflege}

\section{Information}

Bevor eine ältere Person einwilligt, längerdauernd in eine Institution der Langzeitpflege einzutreten, soll sie (und allenfalls ihre Vertrauensperson, ihr Bevollmächtigter in administrativen oder finanziellen Angelegenheiten oder ihr gesetzlicher Vertreter) die Gelegenheit erhalten, die Institution persönlich kennenzulernen, mit einer verantwortlichen Person in der Institution ein Gespräch zu führen und über alle relevanten Informationen (inkl. Reglemente) zu verfügen.

Die Institution soll ihr (bzw. bei Urteilsunfähigkeit ihrer Vertrauensperson oder ihrem gesetzlichen Vertreter) schriftliche Unterlagen abgeben mit gut verständlichen Informationen zu den allgemeinen Aufnahme- und Aufenthaltsbedingungen, zu den Rechten und Pflichten, den Betreuungsmodalitäten und -kosten sowie dem internen und externen Beschwerdewesen. Auch die finanzielle Situation der älteren Person soll angesprochen werden.

\section{Einwilligung}

Nachdem sie die notwendigen Informationen erhalten hat, entscheidet die urteilsfähige, ältere Person selbst über einen Eintritt. Bei Urteilsunfähigkeit ist lediglich ihre Vertrauensperson oder ihr gesetzlicher Vertreter berechtigt, einen solchen Entscheid zu fällen.

Falls es notwendig erscheint, eine ältere Person gegen ihren ausdrücklich geäusserten Willen in eine Institution der Langzeitpflege einzuweisen, soll dies nur nach Rücksprache mit der zuständigen Vormundschaftsbehörde (mittels fürsorgerischer Freiheitsentziehung [FFE]) geschehen.

\section{Regelung finanzieller Angelegenheiten}

Um Interessenkonflikten vorzubeugen, werden das Vermögen und die Einkünfte der älteren 
Person durch sie selbst (oder ihren Bevollmächtigten) und nicht durch die Institution verwaltet. Die Institution sorgt dafür, dass das Personal keine Zuwendungen (auch keine Schenkungen oder Erbschaften) entgegennimmt; ausgenommen bleiben kleine Gelegenheitsgeschenke.

\section{Vertragsauflösung}

Ausser beim Vorliegen gewichtiger Gründe soll die Institution den Vertrag mit einer älteren, pflegebedürftigen Person, die sie aufgenommen hat, später nicht mehr auflösen. Gegebenenfalls hilft die Institution der älteren Person, eine Institution $\mathrm{zu}$ finden, welche sie entsprechend ihrem Gesundheitszustand und ihrer Pflegebedürftigkeit betreuen kann.

\section{Mitglieder der für die Ausarbeitung dieser Richtlinien verantwortlichen Sub- kommission}

Prof. Dr. med. Andreas Stuck, Bern, Vorsitz; Dr. med. Hermann Amstad, Basel (ex officio); Dr. theol. Ruth Baumann-Hölzle, Institut DialogEthik, Zürich; Angeline Fankhauser, Alt-Nationalrätin, Präsidentin VASOS, Oberwil; Prof. Dr. Annemarie Kesselring, Basel; Prof. Dr. iur. Audrey Leuba, Neuenburg; Prof. Dr. med. Charles-Henri Rapin, Genf; Dr. med. Regula Schmitt, Ittigen; Hansruedi Schönenberg, Heimleiter, Zürich; Dr. med. et phil. Urban Wirz, Subingen; Prof. Dr. med. Michel Vallotton, Präsident ZEK, Genf (ex officio).

Die Subkommission dankt folgenden Fachleuten für wertvolle Hinweise

Prof. Lazare Benaroyo, Lausanne; Dr. Georg Bosshard, Zürich; Claudine Braissant, Belmont; Anja Bremi, Zollikon; Dr. Charles Chappuis, Bern; Oskar Diener, Champagne; Werner Egloff, Laupen; Marianne Gerber, Zürich; Prof. Daniel Hell, Zürich; Prof. François Höpflinger, Zürich; Nicolas Kühne, Lausanne; Domenica Schnider Neuwiler, Wil SG; Prof. Hannes Stähelin, Basel; Dr. Markus Zimmermann-Acklin, Luzern; Vérène Zimmermann, Zürich.

Genehmigt vom Senat der SAMW am 18. Mai 2004.

Prof. Michel Vallotton, Genf, Präsident der Zentralen Ethikkommission der SAMW

Der Zentralvorstand des SBK hat diese Richtlinien zur Kenntnis genommen und empfiehlt seinen Mitgliedern und allen Pflegenden, sie anzuwenden und zu achten.

\section{Anhang}

Der Anhang gibt jene Dokumente wieder, die

a) Gesetzescharakter besitzen und je nach Geltungsbereich (Europa, Schweiz, Kantone) zwingend zu beachten sind;

b) als Richtlinien der SAMW gewisse Teilbereiche bei der Behandlung und Betreuung von älteren, pflegebedürftigen Menschen abdecken;

c) weiterführende Literatur zum Thema «Behandlung und Betreuung von älteren, pflegebedürftigen Menschen» enthalten und welche der Subkommission bei der Erarbeitung dieser Richtlinien und Empfehlungen dienlich waren.

\section{a) Rechtliche Grundlagen}

- Übereinkommen zum Schutz der Menschenrechte und der Menschenwürde im Hinblick auf die Anwendung von Biologie und Medizin: Übereinkommen über Menschenrechte und Biomedizin vom 4. April 1997. www.ofj.admin.ch/themen/ bioeth/konvention-biomedizin-d.pdf.

- Schweizerische Bundesverfassung: Art. 7-36 (Grundrechte). www.admin.ch/ch/d/sr/c101.html.

- Bundesgesetz vom 19. Juni 1992 über den Datenschutz. www.admin.ch/ch/d/sr/235_1/index.html.

- Zivilgesetzbuch: Art. 27ff. (Schutz der Persönlichkeit), Art. 360ff. (Vormundschaftsrecht; in Revision). www.admin.ch/ch/d/sr/c210.html.

- Strafgesetzbuch: Art. 115 (Verleitung und Beihilfe zum Suizid), Art. 181 (Nötigung), Art. 320 (Verletzung des Amtsgeheimnisses), Art. 321 (Verletzung des Berufsgeheimnisses). www.admin.ch/ch/d/sr/ 311_0/index2.html.

- Kantonale Gesundheitsgesetze. www.federalism.ch/ documentation/claws/gesundheit/.

- Kantonale Gesetze über Pflegeinstitutionen. www. federalism.ch/documentation/claws/gesundheit/.

- Kantonale Datenschutzgesetze. www.federalism. ch/documentation/claws/.

\section{b) Medizinisch-ethische Richtlinien der Schweizerischen Akademie der Medizini- schen Wissenschaften (www.samw.ch)}

- «Betreuung von Patienten am Lebensende» (2004).

- «Behandlung und Betreuung von zerebral schwerst geschädigten Langzeitpatienten» (2003).

- «Forschungsuntersuchungen am Menschen» (1997).

- «Zwangsmassnahmen in der Medizin» (in Vorbereitung). 


\section{c) Weiterführende Literatur}

- Agence Nationale d'Accréditation et d'Evaluation en Santé. Evaluation des pratiques professionnelles dans des établissements de santé. Limiter les risques de la contention physique de la personne âgée. 2000. www.anaes.fr/publication/geriatrie.

- Arbeitsgruppe «Ethische Richtlinien» des Heimverbandes Schweiz, Fachverband Betagte. Grundlagen für verantwortliches Handeln in Altersund Pflegeheimen. 1997.

- Arbeitsgruppe Gesundheit (AGX) der Schweizerischen Datenschutzbeauftragten. Bericht 2003 betreffend Pflegebedarfsabklärungssysteme (Bewohnerbeurteilungssysteme) in Alters- und Pflegeheimen.

- Association Alzheimer Europe. Directives relatives aux différentes mesures destinées à restreindre la liberté de mouvements. Position paper 2001. www.alzheimer-europe.org.

- Association Alzheimer Europe. Les droits des personnes atteintes de démence. 2001. www. alzheimer-europe.org.

- Association vaudoise d'établissements médicosociaux. Charte éthique. 2001.

- Baumann-Hölzle R. Moderne Medizin - Chance und Bedrohung. Kap. 20: Ethische Probleme in der Geriatrie. Kap. 21: Gelungenes Altwerden und Sterben im Spannungsfeld von Macht und Menschenwürde. Bern, Berlin: Verlag Peter Lang; 2001.

- Comité européen pour la cohésion sociale (CDCS)/Conseil de l'Europe. Améliorer la qualité de vie des personnes âgées en situations de dépendance. 2002. http://book.coe.int.

- European Association for Directors of Residential Care Homes for the Elderly. Charte européenne des droits et libertés des personnes âgées en institution. 1993. www.ede-association.org.

- Forum stationäre Altersarbeit/santésuisse. Konzept zur Einführung eines institutionalisierten Qualitätsmanagements in Schweizer Pflegeheimen gemäss KVG. 1998.

- Gebert A, Kneubühler H-U. Qualitätsbeurteilung und Evaluation der Qualitätssicherung in Pflegeheimen. Bern: Verlag Hans Huber; 2001.
- International Association of Gerontology. The Older Person's Charter of Standards. 1997. www.sfu.ca/iag.

- Kesselring A. Beurteilung der Verhältnismässigkeit von RAI-Items aus pflegewissenschaftlicher Sicht. Institut für Pflegewissenschaft der Universität Basel; 2003.

- Mettner M, Schmitt-Mannhart R. Wie ich sterben will. Patientenautonomie, Selbstverantwortung und Abhängigkeit am Lebensende. Zürich: NZN Buchverlag; 2003.

- Schönenberg HR. Qualitätssicherung und Qualitätsförderung im Langzeitpflegebereich. Managed Care $2000 ;(2): 21-3$

- Schweizerischer Berufsverband für Krankenpflege. Qualitätsnormen für die Pflege und Begleitung von alten Menschen. Bern; 1994.

- Schweizerischer Berufsverband für Krankenpflege et al. Rund um den Heimeintritt: Broschüre für ältere Menschen und ihre Angehörigen. Thun: Ott-Verlag; o. J.

- Schweizerische Gesellschaft für Gerontologie. Richtlinien zur Anwendung freiheitsbeschränkender Massnahmen bei der Behandlung und Pflege betagter Personen. 1999. www.sgg-ssg.ch.

- Schweizerische Sanitätsdirektorenkonferenz. Empfehlungen zum Vollzug des KVG im Bereich der Pflegeheime und der Spitexdienste. 1998.

- Schweizerischer Senioren- und Rentner-Verband. Vorschlag zu einem Kantonalen Gesetz betreffend Heime für alte Menschen und Menschen mit Behinderung. 2001.

- Stuck AE. Multidimensionales geriatrisches Assessment im Akutspital und in der ambulanten Praxis. Schweiz Med Wochenschr 1997;127(43):1781-8.

- United Nations. United Nations Principles for Older Persons. 1999. www.un.org/esa/socdev/iyop/ iyoppop.htm.

- Weltärztebund. Deklaration von Hongkong über die Misshandlung älterer Personen. 1989. www.bundesaerztekammer.de/30/Auslandsdienst/ 99.Handbuch2000.pdf.

- WHO Division of Mental Health. Psychiatry of the elderly - a consensus statement. 1996. www5.who. int/mental health/download.cfm?id=0000000017. 